\title{
Sistema Prolift en la corrección del prolapso genital femenino
}

\author{
Solà Dalenz V, Pardo Schanz J, Ricci Arriola P, Guiloff Fische E. \\ Unidad de Uroginecología y Cirugía Vaginal de Clínica Las Condes. Las Condes, Santiago, Chile.
}

Actas Urol Esp. 2007;31(8):850-857

\begin{abstract}
RESUMEN
SISTEMA PROLIFT EN LA CORRECCIÓN DEL PROLAPSO GENITAL FEMENINO

Objetivo: Revisar la seguridad y eficacia de la corrección de prolapso genital femenino con el nuevo sistema Prolift.

Paciente y Método: Estudio prospectivo de 41 pacientes ingresados para corrección quirúrgica de prolapso anterior y/o posterior genital, entre julio del 2006 y mayo del 2007, en la Unidad de Uroginecología y Cirugía Vaginal de Clínica Las Condes. El periodo de observación fue hasta julio del 2007.

Resultados: Se corrigieron 29 cistoceles: 13 grado II, 14 grado III y 3 grado IV. Treinta rectoceles: 12 grado II, 16 grado III y 3 grado IV. Cinco casos de prolapso de cúpula (malla total, anterior y posterior): 1 grado II y 4 grado IV. El tiempo quirúrgico para Prolift anterior fue de 40 minutos (30 a 50 minutos). Para Prolift posterior 30 minutos (20 a 40 minutos). Para Prolift Total en cúpula 60 minutos ( 40 a 70 minutos). No se registraron complicaciones durante el intraoperatorio. En el postoperatorio inmediato se registró un caso de hematoma perirectal en un paciente con malla posterior para corrección de rectocele. Se trató sólo con anti-inflamatorios. Durante el postoperatorio tardío se registró un caso de erosión vaginal y exposición de $0,5 \mathrm{cms}$ de malla en un caso de prolapso de cúpula, corregido con malla total. Se registraron 2 recidivas asintomáticas en prolapso de cúpula. Una de grado IV a grado III y otra de grado IV, que se presentó como cistocele grado II. La escala visual análoga del dolor en pacientes con Prolift anterior solamente, a las 12 horas 3-4, 24 horas 2 y 48 horas 1. En Prolift posterior solamente, a las 12 horas 4-6, 24 horas 3-4 y 48 horas 1-2. En los casos con Prolift anterior y posterior, y en los casos de prolapso de cúpula, los valores no variaron respecto a los de Prolift posterior. Respecto al grado de satisfacción preguntado durante el control a los 30 días, todas respondieron estar muy satisfechas. La media de seguimiento de las 41 pacientes fue de 7 meses (rango de 2 a 12 meses).

Conclusión: Según nuestra experiencia la corrección del prolapso genital femenino con el Sistema Prolift es seguro y eficaz, sin embargo debemos tener presente que se requiere un seguimiento a largo plazo para registrar el mantenimiento de los buenos resultados.

Palabras clave: Prolapso. Prolift. Gynemesh PS. Cistocele. Rectocele.
\end{abstract}

\section{ABSTRACT}

PROLIFT SYSTEM IN THE CORRECTION OF FEMALE GENITAL PROLAPSE.

Objective: To review the security and effectiveness of female genital prolapse using the new Prolift system.

Patient and Method: Prospective study of 41 patients that have been submitted to correction of cystocele and/or rectocele or vaginal cuff, between July of 2006 and May of 2007 in the Urogynecology and Vaginal Surgery Unit of Clínica Las Condes. The period of observation was completed at July of 2007.

Result: Anterior Prolift mesh was used in 29 cystocele: 13 degree II, 14 degree III and 3 degree IV. Thirty rectocele: 12 degree II, 16 degree III and 3 degree IV. Five cases of vaginal cuff prolapse (total mesh): 1 degree II and 4 degree IV. The media time of surgery was 40 minutes for anterior Prolift (30 to 50 minutes); 30 minutes (20 to 40 minutes) for posterior Prolift and 60 minutes for Total Prolift. They did not present intraoperative complications. During the immediate postoperative time a case of peri-rectal hematoma was observed in a patient with posterior mesh for the rectocele correction. It was treated with oral anti-inflammatory. During the delayed postoperative time a case of vaginal erosion and mesh exposition ( 0.5 centimeters) was observed in a case of vaginal cuff prolapse treated with a Total Prolift. Two asymptomatic recidivate vaginal cuff prolapse were registered; degree IV to III and a case degree IV that was recidivate to cystocele degree II. The visual analogue scale of pain in Anterior Prolift was 3-4 at 12 hours, 2 at 24 hours and 1 at 48 hours. In Posterior Prolift was 4-6 at 12 hours, 3-4 at 24 hours and 1-2 at 48 hours. In cases with Anterior and Posterior Prolift and in Total Prolift the results were the same of Posterior Prolift.

All patients indicated satisfaction in the follow-up at 30 days. The media follow up in the 41 patients was 7 months (range of 2 to 12 months).

Conclusion: According to our experience the correction of the female genital prolapse with Prolift system is safe and effective; nevertheless a long follow-up is required to observe the good results in the time.

Keywords: Genital Prolapse. Prolift. Gynemesh PS. Cystocele. Rectocele. 
$\mathrm{E}$ prolapso genital es una patología que afecta de manera muy importante la calidad de vida de la mujer ${ }^{1}$.Se trata de una patología que afecta a más del 50\% de las multíparas ${ }^{2}$.

Entre los factores de riesgo del prolapso, se encuentra la paridad vaginal ${ }^{3}$, los partos instrumentales (fórceps) ${ }^{4}$, obesidad ${ }^{5}$, neuropatías o denervación ${ }^{6}$, valsalva crónico ${ }^{7}$, alteraciones del tejido conectivo ${ }^{8-10}$, cirugías previas del suelo pélvico $^{3}$, daño de fibras musculares ${ }^{11}$, bajos niveles estrogénicos $^{12}$ y la edad avanzada ${ }^{5}$.

Las técnicas quirúrgicas clásicas poseen un alto porcentaje de recurrencia, que puede ser entre 5 y $40 \%$ para cistocele ${ }^{13,14}$. Estas cifras han llevado a replantearse la fisiopatología del prolapso y a desarrollar nuevas técnicas quirúrgicas. Actualmente las alternativas quirúrgicas aceptadas para la corrección del prolapso son: utilización de material protésico (mallas) ${ }^{15}$, reparación de defectos paravaginales, reparación de defectos pararrectales y reparación del defecto sitio específico.

Las mallas sintéticas se utilizaron por primera vez, como refuerzo de hernias de pared abdominal. Su función es entregar un soporte adicional a los tejidos de mala calidad. La malla ideal debe ser biocompatible, inerte, estéril, no carcinogénica, no debe causar alergias ni rechazo, y debe ser resistente. Actualmente se considera que la mejor malla para el uso en ginecología es la de polipropileno, macroporo, monofilamento. Esta ha sido utilizada como refuerzo de las fascias, con técnica libre de tensión, reduciendo la posibilidad de recidivas.

Un nuevo sistema de malla para la corrección del prolapso, es el sistema Prolift ${ }^{16}$ Se trata de una malla extensa, con sistema de anclaje que permite dar un soporte más integral y que se aplica con técnica de mínima invasión.

Con el objetivo de revisar la seguridad y eficacia de la corrección de prolapso genital femenino con el nuevo sistema Prolift, se aplicó esta técnica en pacientes de nuestra Unidad de Uroginecología y Cirugía Vaginal.

\section{PACIENTE Y MÉTODO}

Se realizó un seguimiento prospectivo de 41 pacientes ingresados para corrección quirúrgica de prolapso anterior y/o posterior genital o prolapso de cúpula, entre julio del 2006 y mayo del 2007, en la Unidad de Uroginecología y Cirugía Vaginal de Clínica Las Condes. Para la presente comunicación, se completó un periodo de observación hasta julio del 2007.

Para ello se utilizó el nuevo sistema de reparación del suelo pélvico, Gynecare Prolift (Johnson \& Johnson, Somerville, New Jersey) (Figs. 1 y 2), El rango de edad de las pacientes fue entre 37 y 90 años, con una media de 54 años. Paridad media de 2 partos vaginales (Tabla 1 ).

A todas las pacientes se les explicó la cirugía a realizar y todas firmaron un consentimiento informado.

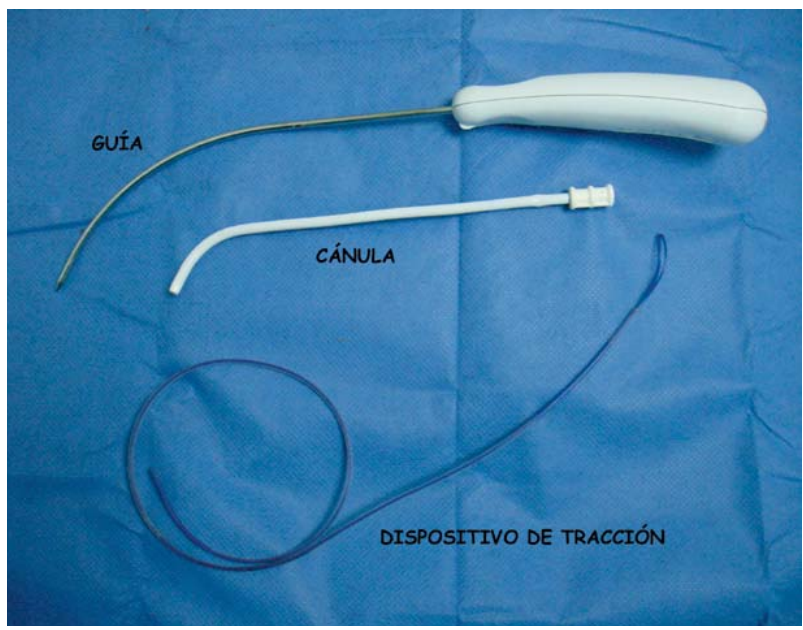

FIGURA 1. Set Gynecare Prolift.

Sistema Gynecare Prolift, compuesto por Malla Gynemesh PS (polipropileno, macroporo, monofilamento), guía, cánula y un dispositivo de tracción.

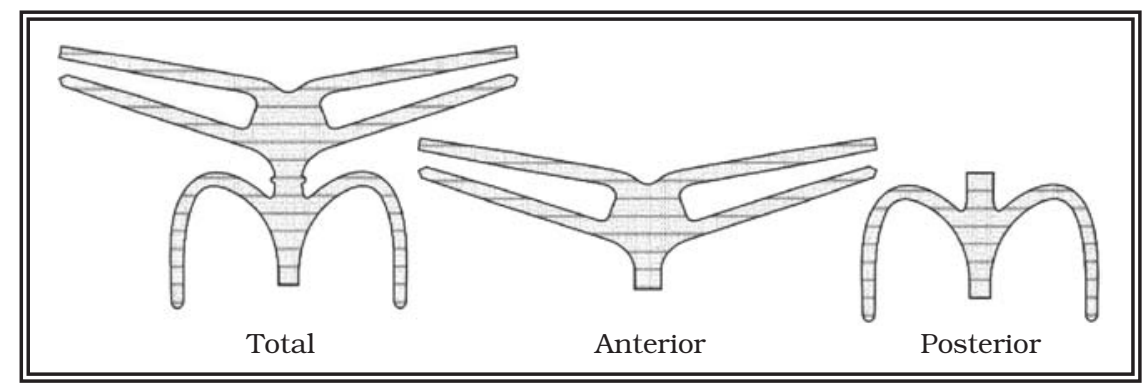

FIGURA 2. Esquema de la malla del Sistema Gynecare Prolift.

Malla de Prolit anterior, posterior y total. La malla total sin cortar se usa en caso de corrección de cistocele y rectocele, con histerectomía asociada o en caso de prolapso de cúpula. 
Tabla 1. Características de las pacientes ingresadas al estudio.

\begin{tabular}{ll}
\hline Total de pacientes en estudio & 41 pacientes \\
Periodo de realización de cirugias & julio del 2006 a mayo del 2007 \\
Periodo de observación & julio del 2006 a julio del 2007 \\
Edad de pacientes & Media 54 años \\
& Rango 37 a 90 años \\
Paridad media & 2 partos vaginales.
\end{tabular}

\section{Criterio de inclusión}

Pacientes con cistocele y/o rectocele o prolapso de cúpula grado II, III o IV según la clasificación POP-Q, que no han sido sometidas a cirugia de corrección por este diagnóstico previamente.

\begin{tabular}{ll} 
Corrección según tipo de prolapso y malla Prolift \\
Cistocele (29): & Prolift Anterior \\
Rectocele (30): & Prolift Posterior \\
Prolapso de Cúpula (5): & Prolift Total \\
\hline
\end{tabular}

\section{Criterio de inclusión}

Pacientes con cistocele y/o rectocele o prolapso de cúpula, grado II, III o IV según la clasificación POP-Q, que no han sido sometidas a cirugía de corrección por este diagnóstico previamente.

En aquellos con incontinencia de orina de esfuerzo o incontinencia potencial, se realizó urodinamia con cistomanometría monocanal, medición de la presión de retro-resistencia uretral (URP) y Q-Tip Test. En todos los casos de prolapso grado III y IV, con potencial aparición de incontinencia una vez corregido con Prolift. Se realizó urodinamia antes de la cirugía, con corrección del defecto, con valva de Bresky.

En la corrección quirúrgica de la incontinencia de orina de esfuerzo, se utilizó TVT ${ }^{17}$ (tipo II+III), TVT-O ${ }^{18}$ o TVT-S ${ }^{19}$ (tipo II).

\section{Preparación preoperatoria de mucosa vaginal}

En las pacientes que se encontraban en menopausia se preparó la mucosa vaginal en forma previa a la cirugía con estriol local por 21 días, debido a que presentaban atrofia.

\section{Profilaxis antibiótica intraoperatoria}

Se utilizó profilaxis antibiótica con cefazolina y metronidazol en tres dosis (cada 8 horas), iniciándola durante la cirugía.

Analgesia del postoperatorio inmediato: Durante el postoperatorio se utilizó analgésicos/anti-inflamatorio por vía oral (ketoprofeno de 50 mg cada 8 horas) y se aplicó la escala visual análoga del dolor a las 12, 24 y 48 horas.

\section{Anestesia}

La anestesia utilizada fue regional espinal.

\section{Alta hospitalaria}

Se dio a las 48 horas en todos los casos.

Control postoperatorio: Se realizó un control a los 14 y 30 días, revisando los resultados de la corrección del prolapso (POP-Q). Además se aplicó un cuestionario de satisfacción.

\section{Descripción del Sistema Prolift}

Este sistema cuenta con implantes de malla y un set de instrumentos (una guía, cánulas y dispositivos de tracción de la malla) para facilitar su introducción y localización (Figs. 1 y 2).

Malla: Se trata de una malla de polipropileno, macroporo, monofilamento, no absorbible (Gynecare Gynemesh PS). Según estas características, cumple con los requisitos de tipo I en la clasificación de mallas protésicas. Esta malla puede ser anterior, posterior o total (Fig. 2).

Malla de Prolift anterior: La malla anterior cuenta con 4 prolongaciones o brazos, para fijarse en el arco tendíneo de la fascia endopélvica (ATFE), utilizando el agujero obturador. Las prolongaciones anteriores de esta malla poseen extremos cuadrados, mientras que las distales tienen extremos triangulares.

Malla de Prolift posterior: La malla posterior posee 2 brazos o prolongaciones para fijarse en el ligamento sacroespinoso por vía tranglútea. Las prolongaciones de esta malla poseen extremos redondeados.

Malla de Prolift total: La malla total es la anterior unida a la posterior formando una sola malla. Es fabricada de esta forma, pero se puede separar cortándola.

Guía: Se trata de un instrumento que permite formar la vía, a través de los tejidos, donde se fijarán las prolongaciones o brazos de los implantes de malla. Está formado por un mango y una aguja con una curva.

Cánula: Instrumento que se introduce para crear el canal por donde pasan los brazos de la malla. Se coloca sobre la guía antes de pasarla y se deja en su lugar después de retirar la guía. Permite el paso de los brazos de la malla y protege el tejido adyacente. 
Dispositivo de tracción: Instrumento para facilitar la colocación y tracción de los brazos de la malla a través de las cánulas. El extremo distal posee un lazo que permite asegurar el brazo de la malla que se desea ajustar.

\section{Uso del Sistema Prolift}

La cánula se ensambla en la aguja de la guía antes de introducirla a través de los puntos previamente marcados en la piel. Una vez situada la cánula se saca la guía. Luego se pasa el dispositivo de tracción a través de la cánula, se engancha 2,5 a $3 \mathrm{~cm}$ del brazo de la malla en el lazo de este dispositivo y se tracciona para exteriorizarlo y así ajustar la malla.

\section{Técnica quirúrgica específica}

Se coloca la paciente en posición ginecológica, con flexión de muslo-abdomen de 90 grados. Vaciamiento de orina vesical con sonda Foley de 16 french.

Sistema Anterior: Se colocan pinzas de Allis en la línea media de la mucosa vaginal, desde la base del cuello vesical hasta un centímetro del fondo de saco anterior. Se infiltra la región submucosa vaginal con solución vasoconstrictora (epinefrina) en dilución 1 a 300.000 , en toda la extensión del prolapso. Incisión medial con bisturí en zonas traccionadas con pinzas de Allis. Disección con tijera de Metzembaum y con presión digital, de la fascia vesico-vaginal, hasta la palpación de ambas espinas ciáticas y espacios paravaginales. Se marca con lápiz en el cruce de línea que nace a nivel del meato urinario y corta con rama ascendente del pubis y borde de membrana obturatriz. Una segunda marca un centímetro hacia fuera y dos hacia abajo desde la primera, a ambos lados. Incisión de $4 \mathrm{~mm}$ con bisturí, en las marcas anteriores. Se coloca valva de Bresky ancha para separar vejiga, se pasa aguja del dispositivo por la primera marca, pasando un centímetro distante del hueso pubiano a través del ATFE, de afuera hacia adentro. Se retira la aguja y se pasa el dispositivo de tracción por la cánula. Se pasa una segunda aguja en la segunda marca, también a través del ATFE, un centímetro proximal a espina ciática. Se repiten ambas maniobras al lado contralateral. Se coloca la malla de Prolift anterior y se ajusta (Figs. 3, 4 y 5).

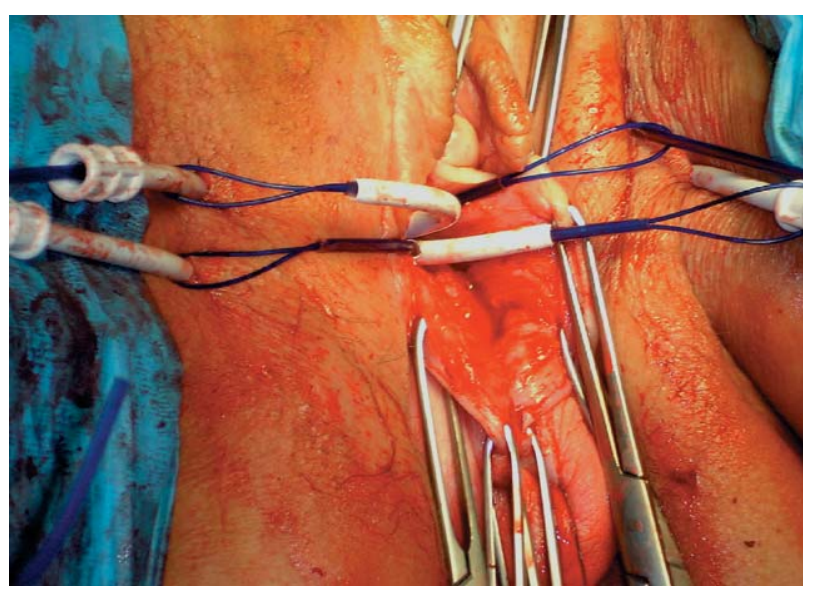

FIGURA 3. Cánula y dispositivo de tracción en aplicación de Prolift anterior.

Cánulas con los dispositivos de tracción en caso de Prolift anterior. Aún no se pone la malla.

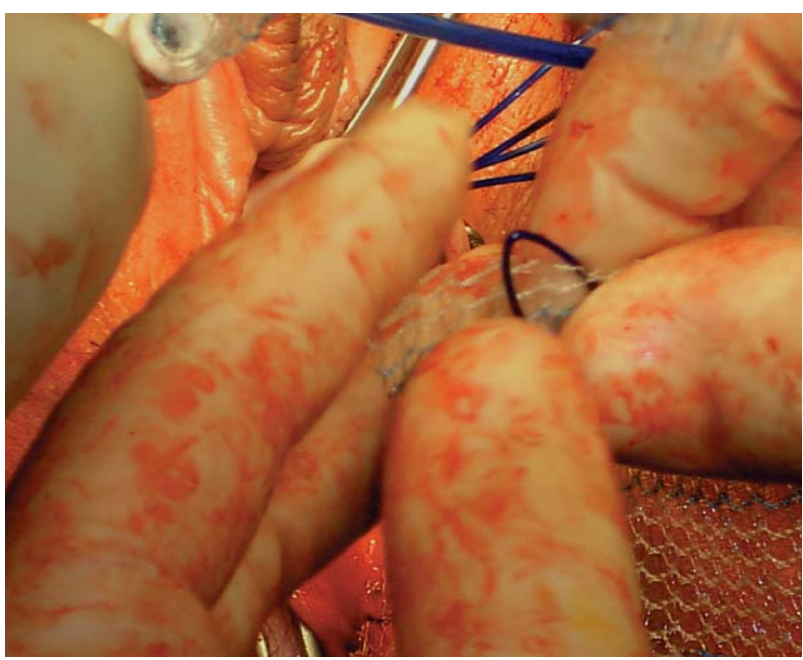

FIGURA 4. Colocación de la malla en el dispositivo de tracción.

Paso de la prolongación o brazo de la malla por el lazo del dispositivo de tracción.

Sistema Posterior: Se marcan dos puntos a tres centímetros por fuera y posterior al ano a cada lado. Se colocan pinzas de Allis en la línea media vaginal, desde el inicio del rectocele hasta $1 \mathrm{~cm}$ del fondo de saco posterior. Se infiltra la región submucosa vaginal con solución vasoconstrictora (epinefrina) en dilución 1 a 300.000, en toda la extensión del prolapso. Incisión medial con bisturi en zonas traccionadas con pinzas de Allis. Disección digital y con tijera de Metzembaum de toda la fascia recto-vaginal, hasta la palpación de ambas espinas ciáticas y espacios pararectales. Se coloca la valva de Bresky ancha para traccionar recto contralateralmente al paso 


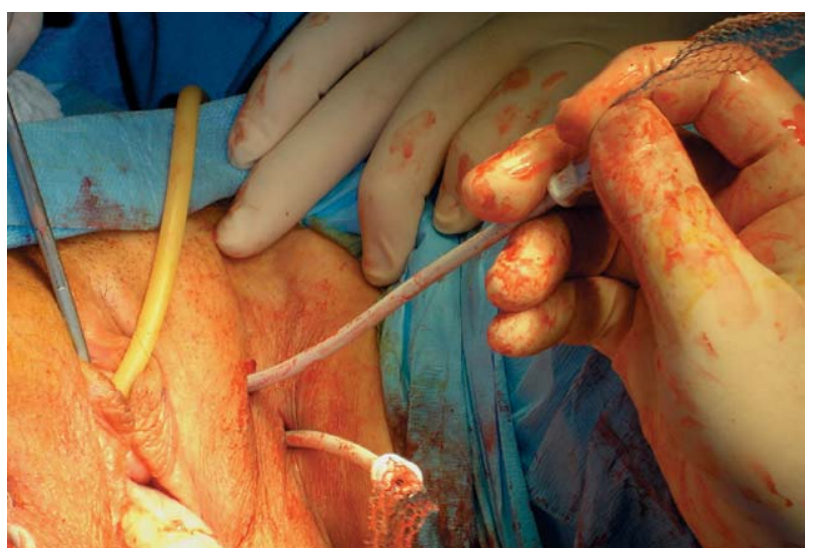

FIGURA 5. Tracción de la malla

Tracción y ajuste de la malla Prolift anterior por medio de los brazos o prolongaciones, a través de las cánulas.

de la aguja de la guía. Se pasa aguja por dicha marca atravesando la grasa perirectal y apoyado por el dedo índice del cirujano, se lleva ésta por debajo de la espina ciática y se atraviesa el ligamento sacroespinos $2 \mathrm{~cm}$ distales a la espina. Se visualiza la aguja y cánula, se retira la primera y se pasa el dispositivo de tracción. Se repite la maniobra contralateralmente. Se coloca la malla de Prolift posterior y se ajusta (Fig. 6).

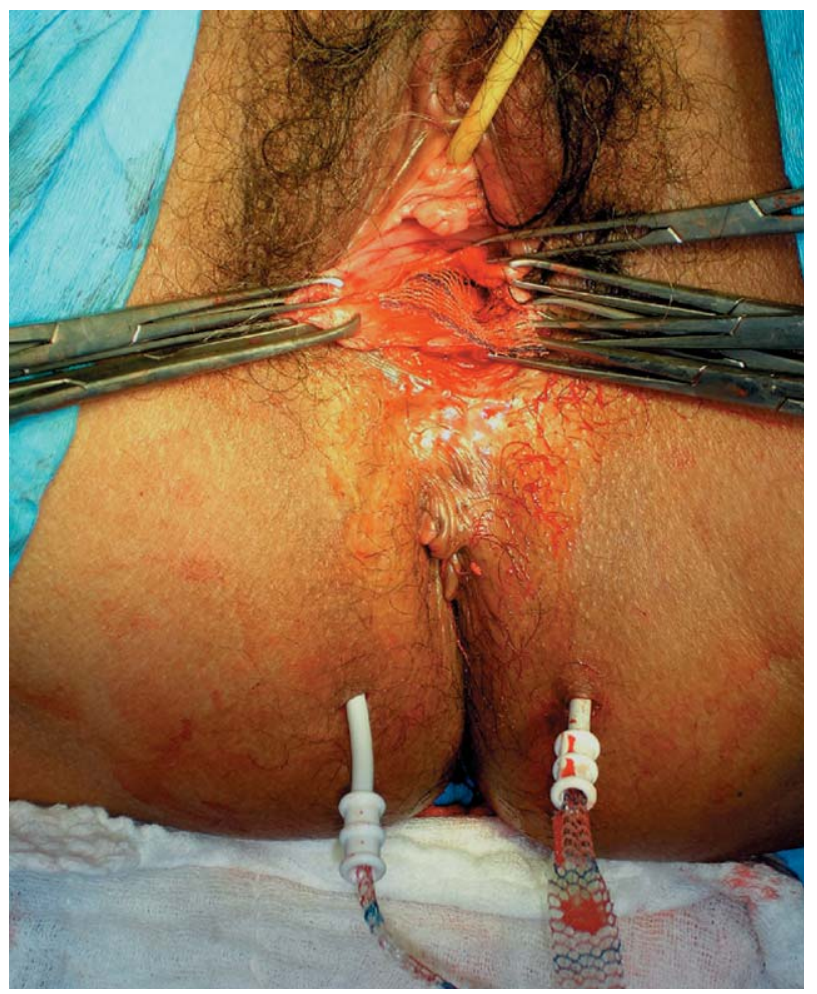

FIGURA 6. Cánula y malla en Prolift posterior.

Malla de Prolift posterior in situ. Aún no se ha suturado la mucosa vaginal sobre la malla.
Los casos de corrección de cistocele se realizaron con Prolift Anterior, los de rectocele con Prolift Posterior y los de cúpula con Prolift Total

En todas las pacientes se registraron las eventuales complicaciones durante los tiempos intraoperatorio y postoperatorio inmediato (hasta las primeras 24 horas), temprano (hasta los 7 días) y tardío (después de los 7 días). Además se registró la presencia de recidiva del prolapso operado

\section{RESULTADOS}

En los 41 pacientes se corrigieron 29 cistoceles: 13 grado II, 14 grado III y 3 grado IV. Treinta rectoceles: 12 grado II, 16 grado III y 3 grado IV. Cinco prolapsos de cúpula (malla total: anterior y posterior): 1 grado II y 4 grado IV. En 6 pacientes se utilizó sólo Prolift anterior (sólo cistocele), en 7 sólo Prolift posterior (sólo rectocele), en 23 ambos (cistocele y rectocele) y en 5 casos la malla total (anterior y posterior) por prolapso de cúpula. El total de mallas Prolift utilizadas fue de 29 anteriores, 30 posteriores y 5 totales (anterior y posterior unidas).

Se realizó cirugía concomitante en 34 pacientes, consistiendo en corrección de incontinencia de orina de esfuerzo con cinta suburetral. Diez TVT por incontinencia tipo II+III, 10 TVT-O por incontinencia tipo II y 14 TVT-S por incontinencia tipo II.

El tiempo quirúrgico de aplicación de Prolift anterior fue de 40 minutos, con rango entre $30 \mathrm{y}$ 50 minutos. El tiempo para Prolift posterior fue de 30 minutos, rango entre 20 y 40 minutos. Para Prolift total en prolapso de cúpula fue de 60 minutos, rango entre 40 y 70 minutos.

No se registraron complicaciones durante el intraoperatorio.

Durante el postoperatorio inmediato se registró un caso de hematoma peri-rectal moderado, en una paciente de 52 años, en que se realizó corrección de rectocele grado II con un Prolift posterior, y se colocó un TVT por incontinencia de orina de esfuerzo tipo II+III. Se realizó tratamiento analgésico y anti-inflamatorio vía oral, con resolución completa, sin necesidad de intervención quirúrgica.

Durante el postoperatorio tardío se presentó un caso de erosión con exposición de la malla hacia la pared vaginal anterior. Correspondió a 
una mujer de 77 años, en que se corrigió un prolapso de cúpula grado IV, con una malla total. La exposición fue de sólo 0,5 centímetros. Se trató con aplicación de estradiol local, sin necesidad de recortar la malla. Esta complicación, se encontró durante el control postoperatorio de los 14 días y era asintomática.

Respecto a la aplicación de la escala visual análoga del dolor en las pacientes que se utilizó Prolift $^{\circledR}$ anterior solamente, a las 12 horas fue 34 , a las 24 horas 2 y a las 48 horas 1 . En las que se utilizó Prolift posterior solamente, a las 12 horas fue 4-6, a las 24 horas fue 3-4 y a las $48 \mathrm{~h}$ fue 1-2. En los casos que se utilizó concomitantemente Prolift anterior y posterior, y en los que se utilizó Prolift Total por prolapso de cúpula, los valores de la escala visual análoga del dolor fueron igual a los de sólo Prolift posterior.

Respecto al grado de satisfacción preguntado durante el control a los 30 días, todas respondieron estar muy satisfechas (Tabla 2).

Tabla 2. Cirugía y resultados.

\begin{tabular}{ll}
\hline Tiempo quirúrgico & \\
Prolift anterior (Cistocele) & \\
Media: & 40 minutos \\
Rango: & 30 a 50 minutos \\
Prolift posterior (Rectocele) & \\
Media: & 30 minutos \\
Rango: & 20 a 40 minutos \\
Prolift Total (Cúpula) & \\
Media: & 60 minutos \\
Rango: & 40 a 70 minutos
\end{tabular}

Complicaciones intraoperatorias

No se presentaron

Complicaciones del post-operatorio inmediato

1 caso de hematoma peri-rectal, tratado sólo con antiinflamatorios, sin necesidad de intervención quirúrgica

\section{Complicaciones del post-operatorio tardio}

1 caso de erosión y exposición de la malla en la cara anterior vaginal

Escala visual análoga de dolor

( 0 a 10. Donde 0 es ningún dolor y 10 es máximo dolor) Tiempo:

$\begin{array}{cccc}\text { Prolift } & \text { Prolift } \\ \text { anterior } & \text { posterior } & \begin{array}{c}\text { Prolift } \\ \text { posterior }\end{array} \\ 12 \mathrm{hrs} & 3-4 & 4-6 & 4-6 \\ 24 \mathrm{hrs} & 2 & 3-4 & 3-4 \\ 48 \mathrm{hrs} & 1 & 1-2 & 1-2\end{array}$

\section{Recidiva}

1 caso de prolapso de cúpula grado IV a III, asintomático. 1 caso de prolapso de cúpula grado IV, recidivado como cistocele grado II.
Se presentaron 2 casos de recidiva en pacientes con corrección de prolapso de cúpula con Prolift total (anterior y posterior formando una sola malla). Ambas recidivas se presentaron asintomáticas. Uno correspondió a una paciente de 81 años, en que recidivó de grado IV a II, y fue observado a los 30 días de control. El otro correspondió a un caso de cúpula grado IV, que a los 3 meses recidivó como cistocele grado III.

La media de seguimiento de las 41 pacientes fue de 7 meses (rango de 2 a 12 meses).

Respecto a la corrección de incontinencia de orina concomitante, en todas se logró corregir la incontinencia de orina de esfuerzo, sin recidiva durante el periodo de observación. No se observaron casos de aparición de incontinencia de orina después de la corrección del prolapso

\section{DISCUSIÓN}

El prolapso genital femenino puede ser entendido como una hernia, es decir hay un caída de un órgano, como puede ser la vejiga o el recto, a través de un defecto de de pared, en este caso el suelo pélvico. Al igual que en la reparación de hernias de pared abdominal, se pueden utilizar mallas como refuerzo o reemplazo de los tejidos. De esta forma se reducirá la posibilidad de una recidiva. Sin embargo, las mallas que se utilizan en la reparación de hernias de pared abdominal, no debieran utilizarse en vagina, ya que aumentan la posibilidad de infección y erosión. La vagina posee condiciones locales especiales y diferentes al resto del organismo, por lo que deben utilizarse mallas especialmente diseñadas para este objetivo. Las mallas quedan bajo la mucosa, no piel, lo que teóricamente aumenta los riegos. Además la vagina ofrece condiciones ideales para desarrollar bacterias potencialmente patógenas. Las mallas a utilizar debieran ser más flexibles. Julián ${ }^{7}$ publicó un éxito de un $100 \%$ en la corrección de cistocele con malla versus un $66 \%$ en casos controles, sólo con colporrafia anterior. Sin embargo, utilizó Marlex, obteniendo un 25\% de complicaciones relacionadas a esta malla, versus un $0 \%$ en los casos de colporrafia anterior sin utilización de malla. En conclusión cualquier malla no da lo mismo, es necesario comprender las características para desarrollar la que más se acerque al ideal, disminuyendo las potenciales 
complicaciones debidas a la utilización de estos materiales que se comportan como prótesis. La malla de este nuevo sistema es la denominada Gynemesh PS ${ }^{\circledR}$. Se trata de una malla de polipropileno, macroporo y monofilamento, lo cual otorga una buena biointegración, con poros que permiten el paso de macrófagos ante la posibilidad del ingreso de una bacteria. Actualmente, en nuestra Unidad, acumulamos una experiencia de 178 casos de uso de malla libre de tensión como refuerzo de fascias en la corrección del prolapso (98 cistoceles y 80 rectoceles con malla) entre noviembre del 2004 y septiembre del 2006 (experiencia próxima a publicar). Sin embargo, con esta nueva experiencia con Prolift, creemos que se da una solución más integral, ya que permite colocar una malla de mayor extensión, que otorga un mejor soporte y refuerzo. Según nuestra opinión creemos que la técnica de Prolift es ideal en la corrección de prolapsos grado III y IV. En los casos de grado II podría ser aceptable un refuerzo con Gynemesh PS con técnica libre de tensión ${ }^{1}$, recortada según el requerimiento, en los casos que se requiera abaratar costos o no se disponga de este nuevo sistema. En este estudio se incluyeron casos de corrección de prolapsos grado II con Prolift, cuyos resultados a futuro podremos comparar con los casos de igual graduación que fueron corregidos con Gynemesh PS libre de tensión, en términos de recidiva del prolapso, función miccional, evacuatoria de heces y sexual. En los seguimientos con Gynemesh PS ha sido informada una reducción de la recidiva del prolapso a un $3 \%^{20}$.

En la experiencia de nuestra práctica quirúrgica con mallas no es habitual la infección y la erosión, lo que también se vio en estos pacientes con Prolift. Esto no sólo es atribuible al tipo de malla que utilizamos, además creemos se debe a algunas medidas que hemos implantado en nuestra práctica habitual en estas cirugías. El protocolo estricto de antibióticos iniciados durante la cirugía y la preparación de la mucosa vaginal en los casos que lo ameritan (atrofia de mucosa en pacientes en postmenopausia) con estriol local por quince días previos y tratamos cualquier infección descubierta durante el control pre-operatorio. De esta forma evitamos la subinfección, que ha quedado demostrado, puede ser causa de erosión y exposición de la malla en vagina. A futuro, una medida que podría reducir estas complicaciones puede ser el uso de mallas que incorporen sustancias antibióticas en sus monofilamentos, recordemos que ya existen suturas que los traen.

Estas pacientes y otras que están incorporándose a diario, serán parte de un seguimiento a largo plazo, lo que sin duda alguna, pretende responder muchas interrogantes sobre el mantenimiento de estos buenos resultados en el tiempo.

Otro punto que debemos mencionar, es la posibilidad de realizar estas cirugias en forma concomitante a cirugias anti-incontinencia con sling. Demostrando que es posible la suma de mallas, sin agregar morbilidad, como lo hemos demostrado en otras experiencias ${ }^{1}$.

Debido a la posibilidad de aparición de incontinencia de orina después de la corrección de un prolapso, nuestro grupo ha incluido como parte de los protocolos de trabajo, el estudio con urodinamia antes de la corrección de casos con prolapsos grado III y IV. De esta manera realizamos urodinamia monocanal con cistomanometría y medición de la presión de retro-resistencia uretral, con corrección del prolapso por medio de una valva Bresky, para evidenciar una potencial aparición de incontinencia después de la corrección de grandes prolapsos. Por esta razón, no es habitual que aparezca incontinencia en los pacientes que operamos con Prolift, tal como queda demostrado en este grupo de pacientes o en otros grupos de paciente corregidos con malla Gynemesh PS recortada según el requerimiento.

\section{CONCLUSIONES}

Según nuestra experiencia la corrección del prolapso genital femenino con el Sistema Prolift es seguro y eficaz, sin embargo debemos tener presente que se requiere un seguimiento a largo plazo para registrar el mantenimiento de los buenos resultados.

\section{REFERENCIAS}

1. Solà V, Pardo J, Ricci P, Guiloff E. Tension Free Monofilament Macropore Polypropilene Mesh (Gynemesh PS) in Female Genital Prolapse Repair. Int Braz J Urol. 2006;32(4):410-415.

2. Carey MP, Dweyer PL. Genital prolapse: vaginal versus abdominal route of repair. Curr Opin Obstet Gynecol. 2001;13:499-505. 
3. Deval B, Rafii A, Poilpot S, Aflack N, Levardon M. Prolapse in the young woman: study of risk factors. Gynecol Obstet Fertil. 2002;30(9):673-676.

4. MacLennan AH, Taylor AW, Wilson DH, Wilson D. The prevalence of pelvic floor disorders and their relationship to gender, age, parity and mode of delivery. BJOG. 2000;107(12):1460-1470.

5. Swift S, Woodman P, O’Boyle A, Kahn M, Valley M, Bland D, Wang W, Schaffer J. Pelvic Organ Support Study (POSST) the distribution, clinical definition, and epidemiologic condition of pelvic organ support defects. Am J Obstet Gynecol. 2005;192(3):795-806.

6. Smith AR, Hosker GL, Warrell DW. The role of partial denervation of the pelvic floor in the aetiology of genitourinary prolapse and stress incontinence of urine. A neurophysiological study. Br J Obstet Gynaecol. 1989;96(1): 24-28.

7. Marinkovic SP, Stanton SL. Incontinence and voiding difficulties associated with prolapse. J Urol. 2004;171(3): 1021-1028.

8. Wong MY, Harmanli OH, Agar M, Dandolu V, Grody MH. Collagen content of nonsupport tissue in pelvic organ prolapse and stress urinary incontinence. Am J Obstet Gynecol. 2003; 189(6):1597-1599; discussion 1599-1600.

9. Goepel C, Hefler L, Methfessel HD, Koelbl H. Periurethral connective tissue status of postmenopausal women with genital prolapse with and without stress incontinence. Acta Obstet Gynecol Scand. 2003;82(7):659-664.

10. Soderberg MW, Falconer C, Bystrom B, Malmstrom A, Ekman G. Young women with genital prolapse have low collagen concentration. Acta Obstet Gynecol Scand. 2004;83(12):1193-1198.

11. Gilpin SA, Gosling JA, Smith AR, Warrell DW. The pathogenesis of genitourinary prolapse and stress incontinence of urine. A histological and histochemical study. $\mathrm{Br} \mathrm{J}$ Obstet Gynecol. 1989;96(1):15-23.

12. Smith P, Heimer G, Norgren A, Ulmstein U. Steroid hormone receptors in pelvic muscles and ligaments in women. Gynecol Obstet Invest. 1990;30(1):27-30.
13. Lucente V, Hale D, Miller D, Madigan J. A Clinical Assesment of Gynemesh PS for the repair of pelvis organ prolapse (POP). Journal of pelvic medicine \& Surgery. 2004;10 Suppl 1:S 35.

14. Julian TM. The efficacy of Marlex mesh in the repair of severe, recurrent vaginal prolapse of anterior midvaginal wall. Am J Obstet Gynecol. 1996;175(6):1472-1475.

15. Solà Dalenz V, Pardo Schanz J, Ricci Arriola P, Guiloff Fische E. Malla de polipropileno monofilamento libre de tensión en la reparación concomitante de cistocele y rectocele. Actas Urol Esp. 2005;29(10):977-980.

16. Baderr G, Fauconnier A, Guyot B, Ville Y. Use of prosthetic materials in reconstructive pelvic floor surgery. An evidence-based analysis. Gynecol Obstet Fertil. 2006;34(4): 292-297.

17. Solà Dalenz V, Pardo Schanz J, Ricci Arriola P, Guiloff Fische E, Chiang Miranda H. TVT associated with other gynecological operations in the same procedure: results and complications. Arch Esp Urol. 2005;58(10):983-988.

18. Solà Dalenz V, Pardo Schanz J, Ricci Arriola P, Guiloff Fische E, Chiang Miranda H. Cirugía mínimamente invasiva en la incontinencia urinaria femenina: TVT-O Actas Urol Esp. 2006;30(1):61-66.

19. Martan A, Masata J, Svabik K TVT SECUR System-tension-free support of the urethra in women suffering from stress urinary incontinence-technique and initial experience. Ceska Gynekol. 2007;72(1):42-49.

20. De Tayrac, Gervaise A, Chauveaud A, Fernandez H. Tension-free polypropylene mesh for vaginal repair of anterior vaginal wall prolapse. J Reprod Med. 2005;50(2):75-80.

Correspondencia autor: P. Ricci Arriola

Unidad de Uroginecología y Cirugía Vaginal de Clínica Las Condes Lo Fontecilla 441, Las Condes, Santiago, Chile

Tel.: (56-2) 2104610

E-mail autor: pricci@vtr.net/pricci@clinicalascondes.cl Información artículo: Original - Urologia femenina

Trabajo recibido: noviembre 2006

Trabajo aceptado: agosto 2007 